\title{
Puerpério de mulheres quilombolas: a luz do cuidado transcultural de Madeleine
}

\section{Leininger}

The puerperium of quilombola women: the light of Madeleine Leininger's transcultural care

El puerperio de las mujeres quilombolas: la luz del cuidado transcultural de Madeleine Leininger

Recebido: 06/06/2021 | Revisado: 15/06/2021 | Aceito: 16/06/2021 | Publicado: 02/07/2021

Tâmara Silva de Lucena

ORCID: https://orcid.org/0000-0002-0480-4458 Universidade Federal de Alagoas, Brasil

E-mail: tamaralucenaa@gmail.com

Jovânia Marques de Oliveira e Silva

ORCID: https://orcid.org/0000-0001-7452-2651

Universidade Federal de Alagoas, Brasil

E-mail: jovaniasilva@gmail.com

Katiane da Silva Mendonça

ORCID: https://orcid.org/0000-0003-3740-9670

Universidade Federal de Alagoas, Brasil

E-mail: katianeksm@gmail.com

Barbara Vitória dos Santos Torres

ORCID: https://orcid.org/0000-0002-0153-1554

Universidade Federal de Alagoas, Brasil

E-mail: barbara.torres@ arapiraca.ufal.br

Vinícius Luiz Farias Oliveira Leandro

ORCID: https://orcid.org/0000-0002-5756-8890

Universidade Federal de Alagoas, Brasil

E-mail: vinicius.leandro@eenf.ufal.br

Ana Carolyna da Silva Rocha

ORCID: https://orcid.org/0000-0001-5538-3895

Universidade Federal de Alagoas, Brasil E-mail: carolynarocha14@gmail.com

Laís Valéria da Silva Bispo

ORCID: https://orcid.org/0000-0001-6150-7698

Universidade Federal de Alagoas, Brasil

E-mail: laisvaleriabispo@hotmail.com

Rita de Cássia de Cerqueira Santos

ORCID: https://orcid.org/0000-0002-3233-9792

Secretaria Municipal de saúde, Brasil

E-mail: ritacerqueira.obstetra@gmail.com

Eli Borges de Freitas Silva

ORCID: https://orcid.org/0000-0002-2332-7407 Universidade Federal de Alagoas, Brasil

E-mail: eli_maceio@hotmail.com

Sueli Terezinha Cruz Rodrigues

ORCID: https://orcid.org/0000-0002-2697-4714

Universidade Federal de Alagoas, Brasil

E-mail: sueli.rodrigues@eenf.ufal.br

Renata Fernandes do Nascimento Rosa ORCID: https://orcid.org/0000-0002-6074-4314

Universidade Federal da Bahia, Brasil E-mail: enfermeirarenatafernandes@gmail.com

Joabson dos Santos Lima ORCID: https://orcid.org/0000-0001-8787-8841 Universidade Federal de Alagoas, Brasil

E-mail: joabsonlima01@gmail.com

Islla Pimentel de Souza

ORCID: https://orcid.org/0000-0002-0703-636X

Universidade Federal de Alagoas, Brasil E-mail: islla-pimentel@hotmail.com

Juliana Alves da Silva

ORCID: https://orcid.org/0000-0001-6857-6100 Universidade Federal de Alagoas, Brasil E-mail: julianaalves130@ hotmail.com 


\author{
Aysla Kalliny dos Reis \\ ORCID: https://orcid.org/0000-0002-1649-2156 \\ Universidade Federal de Alagoas, Brasil \\ E-mail: ayslla_kaliny@hotmail.com
}

\begin{abstract}
Resumo
Objetivo: Analisar os fatores que influenciam o cuidado de mulheres quilombolas no puerpério. Método: Trata-se de um estudo de natureza qualitativa, embasado na etnoenfermagem e no referencial teórico da Teoria da Diversidade e Universalidade do Cuidado de Madeleine Leininger, com o objetivo de descrever o cuidado de mulheres quilombolas no período pós-parto salientando os cuidados culturais e religiosos da comunidade. Resultados: As mulheres quilombolas utilizam de conhecimentos culturais, empíricos e religiosos para realizar a assistência em saúde. Ao utilizarem as plantas, sabem para que estão utilizando, pois especificam que tipo de planta é, bem como a sua indicação, é o que se observa na fala de uma das entrevistadas a seguir, se faz uso também de rezas, curas e banhos de sol para auxiliar no processo de recuperação. Conclusão: Os estudos sobre os diferentes tipos de cuidado e a medicina natural, juntamente com os fatores que os engloba, permite uma melhor visão da circunspecção da identidade cultural e dos conhecimentos empíricos atribuídos aos diferentes públicos assistidos pelos profissionais de saúde.
\end{abstract}

Palavras-chave: Afrodescendente; Período pós-parto; Assistência integral à saúde; Enfermagem.

\begin{abstract}
Objective: To analyze the factors that influence the care of quilombola women in the puerperium. Method: This is a qualitative study, based on ethno-nursing and the theoretical framework of Madeleine Leininger's Theory of Diversity and Universality of Care, with the objective of describing the care of quilombola women in the postpartum period, emphasizing cultural care and religious in the community. Results: Quilombola women use cultural, empirical and religious knowledge to provide health care. When using the plants, they know what they are using it for, because they specify what type of plant it is, as well as its indication, it is what is observed in the speech of one of the interviewees below, it also makes use of prayers, cures and baths. sun to assist in the recovery process. Conclusion: Studies on different types of care and natural medicine, together with the factors that encompass them, allow a better view of the circumspection of cultural identity and empirical knowledge attributed to the different audiences assisted by health professionals.
\end{abstract}

Keywords: African continental ancestry group; Postpartum period; Comprehensive health care; Nursing.

\title{
Resumen
}

Objetivo: Analizar los factores que influyen en el cuidado de las mujeres quilombolas en el puerperio. Método: Este es un estudio cualitativo, basado en la etnoenfermería y el marco teórico de la Teoría de la diversidad y la universalidad de la atención de Madeleine Leininger, con el objetivo de describir el cuidado de las mujeres quilombolas en el período posparto, haciendo hincapié en la atención cultural. y religiosos en la comunidad. Resultados: Las mujeres quilombolas utilizan el conocimiento cultural, empírico y religioso para brindar atención médica. Cuando usan las plantas, saben para qué lo usan, porque especifican qué tipo de planta es, así como su indicación, es lo que se observa en el discurso de uno de los entrevistados a continuación, también hace uso de oraciones, curas y baños. sol para ayudar en el proceso de recuperación. Conclusión: Los estudios sobre diferentes tipos de atención y medicina natural, junto con los factores que los abarcan, permiten una mejor visión de la circunspección de la identidad cultural y los conocimientos empíricos atribuidos a los diferentes públicos atendidos por los profesionales de la salud.

Palabras clave: Grupo de ascendencia continental africana; Período posparto; Atención integral de salud; Enfermería.

\section{Introdução}

A grande diversidade de espécies do bioma brasileiro é considerada uma das mais importantes fontes de princípio ativo do planeta (Sousa et al., 2017). A fitoterapia e o uso de plantas medicinais fazem parte da prática da medicina popular, constituindo um conjunto de saberes internalizados nos diversos usuários e praticantes, especialmente pela tradição oral (Bruning, Mosegui \& Vianna, 2012).

A contribuição dos povos afrodescendentes é de extrema importância para a formação da cultura brasileira. Esses povos são em parte representados pelas comunidades remanescentes de quilombos, que mantêm costumes e conhecimentos sobre utilização e manejo dos recursos vegetais, principalmente os fitoterápicos, devido ao baixo custo e ao fácil acesso da população à flora nativa e naturalizada (Gomes \& Bandeira, 2012; Barbosa et al., 2020). Os quilombos podem ser descritos como comunidades autônomas de escravos fugitivos, sendo uma organização social da população negra em cenário de resistência, buscando o resgate de sua cultura (Brasil, 2013). 
Nessas localidades a assistência à mulher no ciclo gravídico-puerperal era realizada por outras mulheres, sejam as mais velhas, experientes e parteiras, que desenvolviam uma relação de proximidade com a parturiente por compartilharem suas próprias histórias de vida, fundamentadas nos conhecimentos que adquiriram através das experiências passadas de geração a geração e técnicas empíricas (Bonfim et al., 2018).

Madeleine Leininger, em sua Teoria Transcultural, propõe uma visão que permite ao enfermeiro planejar junto com a pessoa assistida os cuidados necessários, preservando as características culturais e religiosas às quais o grupo pertence (Siqueira et al., 2018).

$\mathrm{O}$ fato de ter poucos estudos sobre essa parcela da população demonstra a necessidade da pesquisa para as áreas da saúde, em especial a enfermagem, a qual atua diretamente com essas mulheres e percebe a dificuldade em relação ao aspecto cultural nessa interação com a puérpera quilombola. Partindo dessas considerações, o presente estudo teve como objetivo analisar os fatores que influenciam o cuidado de mulheres quilombolas no puerpério.

\section{Metodologia}

Trata-se de um estudo de natureza qualitativa, o qual possibilita a real compreensão do significado e da intencionalidade que permeia a sociedade, como os atos, as relações e as estruturas sociais humanas (Greinert, Carvalho, Capel, Marques \& Milani, 2018). Ainda, o estudo foi embasado na etnoenfermagem e no referencial teórico da Teoria da Diversidade e Universalidade do Cuidado de Madeleine Leininger, com o objetivo de descrever o cuidado de mulheres quilombolas no período pós-parto.

A coleta de dados se deu por meio de entrevista com roteiro semiestruturado elaborado pelas pesquisadoras. Pretendese descrever como são as práticas de utilização de plantas medicinais baseadas na cultura local das mulheres da Comunidade de Remanescente Quilombola Muquém.

Foi realizado na Comunidade de Remanescente Quilombola Muquém que está situada na zona rural do Município de União dos Palmares/AL. O estudo teve como critérios de inclusão: mulheres que já vivenciaram o período puerperal e que nasceram na Comunidade de Remanescente Quilombola Muquém. E como critério de exclusão: menores de 18 anos e/ou inexistência de capacidade cognitiva que impossibilita a comunicação. Os princípios éticos foram seguidos em todas as fases do estudo.

Os princípios éticos foram seguidos em todas as fases do estudo. Assim, o projeto foi encaminhado à Secretaria Municipal de Saúde de União dos Palmares para autorização da realização do estudo através do consentimento do diretor responsável, bem como da líder da Comunidade Remanescente Quilombola Muquém. Por conseguinte, o estudo foi submetido ao Comitê de Ética em Pesquisa com Seres Humanos (CEP) através da Plataforma Brasil, e foi aprovado sob parecer $\mathrm{n}^{\circ}$ 2.725.381, em 20 de junho de 2018 (Brasil, 2012), obedecendo às normas que regem a Resolução n 466/12 do Ministério da Saúde e a Resolução 510/2016 (Brasil, 2016). Em seguida o projeto foi encaminhado à Secretaria Municipal de Saúde de União dos Palmares, para autorização da realização do estudo no território quilombola.

Após aprovado, os entrevistados foram convidados para participar da pesquisa de forma voluntária e sem receber nenhum valor em espécie. Houve uma prévia apresentação do estudo as mulheres quilombolas que já vivenciaram o período pósparto, sendo solicitado às mesmas a assinatura em duas vias do Termo de Consentimento Livre e Esclarecido (TCLE), onde, uma via permanecerá com ela, a fim de informá-la sobre o objetivo do estudo, método e procedimentos, garantindo seu anonimato e assegurando-o quanto ao seu direito de retirar seu consentimento a qualquer momento e, a outra via ficará com a pesquisadora. Em suma, ratifica-se o respeito à confidencialidade dos sujeitos do estudo e o compromisso com a pesquisa. 


\section{Resultados}

As mulheres quilombolas utilizam de conhecimentos culturais, empíricos e religiosos para realizar a assistência em saúde. Ao utilizarem as plantas, sabem perfeitamente para que estão utilizando, pois especificam que tipo de planta é, bem como a sua indicação, é o que se observa na fala de uma das entrevistadas a seguir.

"Vou dizer a senhora, era de sene, cebola branca, era piranha. Era essas três coisas, somente três goles, era um purgante pra gente. Mas a gente ficava na moda, tudo limpinha, não ficava nada, saia tudo o que tinha lá dentro. Aí pronto, a gente era saudável nessa época. (Amara) Agora, eu tomava tanto chá de mato que a minha tia fazia. Por que quando a pessoa ganha menino no hospital a pessoa tem que tomar chá para limpar tudo por dento, barbatimão que é bom... Cerveja preta, que é pra limpar o sangue por dentro, por que lá no hospital eles só tiram o menino, não dão nada pra limpar por dentro. Bebi uma garrafa de água inglesa também $3 x$ por dia pra sair aquele sangue pisado. $O$ meu saia tanto, saia cada taiada... se não beber nada pra limpar aquilo fica por dentro matando. Eu tinha até medo quando ia fazer xixi!”

Nesse diapasão, percebe-se que o conhecimento empírico é repassado entre as gerações, ou seja, de mãe para filha, de tia para sobrinha; e isso contribui para que a utilização de plantas medicinais com fins curativos se propague até os dias atuais na comunidade. As mulheres ao relatarem que os chás "limpavam tudo por dentro" estão enfatizando que para elas se ficassem restos placentários ou se houvesse a formação de coágulos estes chás eram capazes de fazer toda esta limpeza, prevenindo que elas tivessem qualquer infecção. Quando se referiam que se "lavavam por baixo", estão referindo-se à utilização das plantas medicinais para fazer banho de assento com o objetivo de possibilitar a cicatrização e evitar a infecção.

Além disso, as mulheres do Muquém utilizam-se de rezas como meio para obter a cura espiritual, física e emocional, pois acreditam que as dores de cabeça com que são acometidas no pós parto podem ser cessadas por meio desta prática. Esta proposição pode-se observar na seguinte fala:

"Eu mandei a menina curar, a cura de sol e sereno, melhorei um pouco, mais ainda sinto dor de vez em quando. A cura é quando ela reza na sua cabeça."

Conforme a crença da curandeira da comunidade quilombola expressou em sua fala que as mulheres devem guardar resguardo após o parto sem se expor ao sol e sereno.

"Só se ver pedindo pra curar a cabeça, eu rezo... Mas ali foi juntando na cabeça o sol e sereno. Hoje em dia as mulheres é tudo com dor de cabeça, aquilo ali é o resguardo que não cuidou, levava sol, sereno, aí depois fica com essa dor de cabeça, prejudicada."

A prática de rezar realizada pelas curandeiras da comunidade do Muquém é uma forma de dar significado ao cuidado cultural naquele contexto.

\section{Discussão}

A Política Nacional de Plantas Medicinais e Fitoterápicos (PNPMF) regulamentada por meio do Decreto $\mathrm{n}^{\circ}$ 5.813, de 22 de junho de 2006, reconhece a utilização da fitoterapia no cotidiano da população brasileira, aliada ao fato de o Brasil ser o país que detém a maior parcela da biodiversidade mundial, em torno de 15 a 20\% (Brasil, 2006). 
A utilização das plantas medicinais está orientada nas influências culturais dos colonizadores europeus, indígenas e africanos e, ainda, pelo conhecimento popular desenvolvido por grupos que convivem com a natureza, observando-a e explorando suas potencialidades, mantendo vivo e crescente o conhecimento popular, por tanto a realização de pesquisas nas comunidades tradicionais, acerca da utilização das plantas medicinais é de fundamental importância para a obtenção de características específicas do local e da região em que estas se encontram (Carneiro et al., 2020)

Estudos apontam que as parteiras da Comunidade de Barra do Ararapira utilizavam chá de pé-de-galinha, de sene e de abútua ou vinho quente para a menstruação descer (Muniz \& Silva, 2016) Nota-se, que elas partilhavam do mesmo pensamento das mulheres quilombolas do Muquém, que tomavam os chás, a cerveja preta ou a água inglesa para atingir os mesmos resultados: evitar que o sangue coagulasse, pois para elas isso levaria ao adoecimento. Entretanto, na literatura, o uso da cerveja preta está sempre associado ao efeito galactogênico, sendo um dos métodos não-farmacológicos mais difundido, até mesmo entre profissionais de saúde (Brotto et al., 2015).

A religiosidade é outro ponto muito relevante nessas comunidades, aparecendo como pano de fundo, de forma que as mães buscavam a Deus quando da ocorrência do agravo de saúde principalmente ligado com suas crianças, almejando tanto a resolução do problema como uma orientação divina acerca do modo como deveriam proceder (Siqueira, Jesus \& Camargo, 2016).

Ainda, no âmbito das mulheres quilombolas, as parteiras eram as responsáveis por preparar o banho com as plantas medicinais para a mulher no pós-parto, dentre as plantas utilizadas no banho de assento estavam, aroeira, quixabeira, umbuzeiro e umburana que funcionavam como anti-inflamatório na higiene da genitália externa (Bonfim et al., 2018 como citado em Lucena, 2019).

Os profissionais devem compreender, reconhecer e valorizar as práticas populares e tradicionais em saúde que contribuam para a saúde e o bem-estar biopsicossocial da comunidade, familiarizando-se e aprendendo a lidar com elas para o cuidado da família; adotando condutas ativas e produtivas na comunidade com o objetivo de desmistificar alguns assuntos e de promover a saúde da população (Oliveira et al., 2006).

A sobreposição de saberes populares deve ser manejada adequadamente pelo profissional de saúde, que deve conhecêla de forma científica e avaliar a efetividade para a assistência, essa sobreposição é notada quando o usuário não realiza o tratamento considerado como "convencional" baseado em dados científicos mas em contrapartida, utiliza tratamentos alternativos por se aproximarem de suas necessidades específicas e por fazerem parte de sua cultura (Junges, Barbiani, Ávila, Fernandes \& Lima, 2011).

Os profissionais de saúde precisam planejar os seus cuidados tanto no viver coletivo, quanto nas individualidades, garantindo a dignidade para cada indivíduo. Ainda, nota-se um grande desafio de troca de saberes entre os profissionais que atendem comunidades quilombolas e os saberes e as práticas tradicionais dos integrantes das comunidades, fazendo-se necessário que os profissionais adequem/adaptem os seus serviços conforme as características das comunidades, através da valorização dos relacionamentos, desenvolvimento de escuta qualificada, trabalho multidisciplinar e projetos coletivos, com estabelecimento de parcerias e compromissos com as pessoas, respeitando seus modos de vida e sua família, garantindo assim, uma melhor qualidade no cuidado (Schek, Ianiski, Rzigoski, Vontroba \& Mix, 2020; Santos \& Nascimento, 2019).

Ressalta-se também a importância da inclusão nas universidades, acerca de práticas baseadas em evidências científicas que possam contribuir na redução de técnicas utilizadas como rotina ou embasá-las em dados científicos (Vieira et al., 2019). Ainda nesse contexto o currículo deve contemplar a abordagem de temas transversais que envolvam conhecimentos, vivências e reflexões sistematizadas sobre os direitos humanos, educação e cultura das relações étnico-raciais e história da cultura afrobrasileira, o que vai de encontro com o cuidado transcultural de Madeleine Leininger (Freitas-Júnior et al., 2019). 


\section{Considerações Finais}

Os estudos sobre os diferentes tipos de cuidado e a medicina natural, juntamente com os fatores que os engloba, permite uma melhor visão da circunspecção da identidade cultural e dos conhecimentos empíricos atribuídos aos diferentes públicos assistidos pelos profissionais de saúde.

Como resultado do presente estudo, pode-se destacar a identidade cultural da comunidade quilombola, presente no distrito de Alagoas, essa que por sua traz particularidades frente ao período puerperal da mulher, conhecidas como fundamentais, mediante as experiências e ensinamentos de seus antecessores.

É notória a importância da preservação das identidades de cada grupo social, de tal maneira que o profissional da saúde possa adequar-se ao melhor tipo de cuidado, sendo esse respeitoso e de qualidade, a fim de preservar o direito de cada assistido.

Ademais, se faz necessário novos estudos acerca dessa temática, para conhecer melhor os cuidados com a saúde realizados nessas comunidades, de forma que esses conhecimentos possibilitem uma assistência de saúde mais assertiva e humanizada, atrelando os conhecimentos socioculturais com os conhecimentos teóricos científicos, a fim de melhorar os cuidados realizados nas comunidades e fortalecer vínculos.

\section{Referências}

Barbosa, K. A., Souza, L. F., Silva, F. G., Vitorino, L. C., Bessa, L. A., Menino, G. C. O., \& Bretas, M. L. B. (2020). Etnobotânica Quilombola: um estudo de caso em uma comunidade de descendentes de escravos do centro do bioma Cerrado. Research, Society and development, 9(8):e332985797. https://rsdjournal.org/index.php/rsd/article/view/5797/4865

Bonfim, J. O., Prado, I. F., Sorte, E. T. B., Couto, P. L. S., França, N. M., Gomes, A. M. T. (2018). Práticas de cuidado de parteiras e mulheres quilombolas à luz da antropologia interpretativa. Revista Brasileira em Promoção da Saúde, 31(3):1-11. https://periodicos.unifor.br/RBPS/article/view/7081/pdf

Brasil. Conselho Nacional de Saúde. (2012) Resolução n 466, de 12 de dezembro de 2012. Dispõe sobre diretrizes e normas regulamentadoras de pesquisas envolvendo seres humanos. Diário Oficial da República Federativa do Brasil, Brasília, DF. http://conselho.saude.gov.br/resolucoes/2012/Reso466.pdf

Brasil. Conselho Nacional de Saúde (2016). Resolução nº 510, de 07 de abril de 2016. Dispõe sobre as normas aplicáveis a pesquisas em Ciências Humanas e Sociais. Diário Oficial da República Federativa do Brasil, Brasília, DF. http://conselho.saude.gov.br/resolucoes/2016/Reso510.pdf

Brasil. Ministério da Saúde. (2006). Política nacional de plantas medicinais e fitoterápicos. Secretaria de Ciência, Tecnologia e Insumos Estratégicos, Departamento de Assistência Farmacêutica. Brasília, DF: Ministério da Saúde. http://bvsms.saude.gov.br/bvs/publicacoes/politica_nacional_fitoterapicos.pdf

Brasil. Ministério da Saúde. (2013). Política Nacional de Saúde Integral da População Negra: uma política do SUS. Secretaria de Gestão Estratégica e Participativa, Departamento de Apoio à Gestão Participativa. 2. ed. Brasília, DF: Editora do Ministério da Saúde. http://bvsms.saude.gov.br/bvs/publicacoes/politica_nacional_saude_integral_populacao.pdf

Brotto, L. D. A., Marinho, N. D. B., Miranda, I. P., Lima, E. F. A., Leite, F. M. C., \& Primo, C. C. (2015). Uso de galactogogos no manejo da amamentação: revisão integrativa da literatura. Revista de pesquisa cuidado é fundamental, 7(1):2169-2180. https://www.redalyc.org/pdf/5057/505750945040.pdf

Bruning, M. C. R., Mosegui, G. B. G., \& Vianna, C. M. M. (2012). A utilização da fitoterapia e de plantas medicinais em unidades básicas de saúde nos municípios de Cascavel e Foz do Iguaçu - Paraná: a visão dos profissionais de saúde. Ciência \& Saúde Coletiva, 17(10):2675-2685. http://www.scielo.br/scielo.php?script=sci_arttext\&pid=S1413-81232012001000017\&lng=en

Carneiro, V. P. P., Guny, M. P., Otênio, J. K., Menetrier, J. V., Medeiros, K. A., Bonkoski, V. R., Gasparotto Junior, A., Estevan, D. A., Castro, T. E., Lourenço, E. L. B., Velasquez, L. G., \& Jacomassi E. (2020). Perfil do uso de plantas medicinais por moradores da área rural de um Município do Estado do Paraná. Research, Society and Development, 9(10):e5099108710. http://dx.doi.org/10.33448/rsd-v9i10.8710

Freitas-Júnior, R. A. O., Santos, C. A. D., Lisboa, L. L., Freitas, A. K. M. S. O., Garcia, V. L., \& Azevedo, G. D. (2019). Incorporando a Competência Cultural para Atenção à Saúde Materna em População Quilombola na Educação das Profissões da Saúde. Revista Brasileira de Educação Médica, 42(2):99-109. https://www.scielo.br/pdf/rbem/v42n2/0100-5502-rbem-42-02-0100.pdf

Gomes, T. B., \& Bandeira F. P. S. F. (2012). Uso e diversidade de plantas medicinais em uma comunidade quilombola no Raso da Catarina, Bahia. Acta Botanica Brasilica, 26(4):796-809. http://www.scielo.br/scielo.php?script=sci_arttext\&pid=S0102-33062012000400009\&lng=en

Greinert, B. R. M., Carvalho, E. R., Capel, H., Marques, A. G., \& Milani, R. G. (2018). A relação mãe-bebê no contexto da depressão pós-parto:estudo qualitativo. Saúde e Pesquisa, 1(1):81-88. https://periodicos.unicesumar.edu.br/index.php/saudpesq/article/view/5919/3168

Junges, J. R., Barbiani, R., Ávila, S. N., Fernandes, R. B. P., \& Lima, M. S. (2011). Saberes populares e cientificismo na estratégia saúde da família: complementares ou excludentes?. Ciência \& Saúde Coletiva, 16(11):4327-4335. http://www.scielo.br/scielo.php?script=sci_arttext\&pid=S1413$81232011001200005 \& \operatorname{lng}=\mathrm{en}$.

Lucena, T. S. (2019). Práticas de cuidado de puérperas quilombolas à luz da teoria transcultural (Dissertação de pós-graduação). Universidade Federal de Alagoas - UFAL, Maceió, AL, Brasil. http://www.repositorio.ufal.br/handle/riufal/4998 
Research, Society and Development, v. 10, n. 7, e56210716877, 2021

(CC BY 4.0) | ISSN 2525-3409 | DOI: http://dx.doi.org/10.33448/rsd-v10i7.16877

Muniz, J. C., \& Silva, L. E. (2016). "Mais que isso eu não posso falá”: notas sobre benzeduras e parteiragens Caiçara em Guaraqueçaba/PR. Revista Brasileira de Geografia Médica e da Saúde, 12(23):31-43. http://www.seer.ufu.br/index.php/hygeia/article/view/33704

Oliveira, A. T. S. A., Moreira, C. T., Machado, C. A., Vasconcelos-Neto, J. A., \& Machado, M. F. A. S. (2006) Crendices e práticas populares: influência na assistência de enfermagem prestada à criança no programa saúde da família. Revista Brasileira em Promoção da Saúde, 19(10):1118https://www.redalyc.org/articulo.oa?id=40819104

Santos, A. N. S., \& Nascimento, E. R. (2019). Proposições de cuidado cultural à enfermagem frente a aspectos da saúde reprodutiva de mulheres quilombolas. Revista Baiana de Enfermagem, 33:e33375. https://cienciasmedicasbiologicas.ufba.br/index.php/enfermagem/article/view/33375/20404

Schek, G., Ianiski, F. R., Rzigoski, D., Vontroba, A., \& Mix, P. R. (2020). Cuidados de uma comunidade remanescente de quilombolas à luz da teoria transcultural de Madeleine Leininger. Revista saúde, 14(3-4):71-78. http://revistas.ung.br/index.php/saude/article/view/4327/3200

Siqueira, S. M. C., Jesus, V. S., \& Camargo, C. L. (2016). Itinerário terapêutico em situações de urgência e emergência pediátrica em uma comunidade quilombola. Ciência \& Saúde Coletiva, 21(1):179-189. http://www.scielo.br/scielo.php?script=sci_arttext\&pid=S1413-81232016000100179\&lng=pt.

Siqueira, S. M. C., Jesus, V. S., Santos, L. F. N., Muniz, J. P. O., Santos, E. N. B., \& Camargo, C. L. (2018). Percepções de urgência e emergência pediátrica entre quilombolas: uma abordagem à luz de Leininger. Revista Enfermagem UERJ, 26(1):e21492. http://docs.bvsalud.org/biblioref/2018/11/948421/percepcoesde-urgencia.pdf

Sousa, I. J. O., Araújo, S., Negreiros, P. S., França, A. R. S., Rosa, G. S., Negreiros, F. S., \& Gonçalves, R. L. G (2017). A diversidade da flora brasileira no desenvolvimento de recursos de saúde. Revista uningá review, 31(1):35-39. http://revista.uninga.br/index.php/uningareviews/article/view/2048/1640

Vieira, S. N., Vidigal, B. A. A., Sousa, A. M., Reis, L. N., Teixeira, E., \& Vasconcelos, M. N. G. (2019). Violência obstétrica: convergências e divergências entre acadêmicos de enfermagem e medicina. Enfermagem em Foco, 10(6):21-27. http://revista.cofen.gov.br/index.php/enfermagem/article/view/2068/646 